\title{
Evidence for the duplex theory of tactile texture perception
}

\author{
MARK HOLLINS and S. RYAN RISNER \\ University of North Carolina, Chapel Hill, North Carolina
}

\begin{abstract}
Three experiments are reported bearing on Katz's hypothesis that tactile texture perception is mediated by vibrational cues in the case of fine textures and by spatial cues in the case of coarse textures. Psychophysical responses when abrasive surfaces moved across the skin were compared with those obtained during static touch, which does not provide vibrational cues. Experiment 1 used two-interval forced-choice procedures to measure discrimination of surfaces. Fine surfaces that were readily discriminated when moved across the skin became indistinguishable in the absence of movement; coarse surfaces, however, were equally discriminable in moving and stationary conditions. This was shown not to result from any inherently greater difficulty of fine-texture discrimination. Experiments 2 and 3 used free magnitude estimation to obtain a more comprehensive picture of the effect of movement on texture (roughness) perception. Without movement, perception was seriously degraded (the psychophysical magnitude function was flattened) for textures with element sizes below $100 \mu \mathrm{m}$; above this point, however, the elimination of movement produced an overall decrease in roughness, but not in the slope of the magnitude function. Thus, two components of stimulation (presumably vibrational and spatial) contribute to texture perception, as Katz maintained; mechanisms for responding to the latter appear to be engaged at texture element sizes down to $100 \mu \mathrm{m}$, a surprisingly small value.
\end{abstract}

In his classic treatise The World of Touch, David Katz (1925/1989) advanced the view that the tactile perception of the texture of surfaces is a complex process, depending on a "spatial sense" for discernment of coarse textures and a "vibration sense" for an appreciation of finer textures. Katz offered a number of ingenious observations to support his theory: For example, he demonstrated that papers can be discriminated by an observer drawing a wooden rod across them but that wrapping the rod in cloth greatly impairs performance (p. 115). These results suggest the use of vibrational cues. Katz's view, referred to here as the duplex theory of tactile texture perception, is succinctly captured in his statement that surfaces touched very lightly could not be clearly perceived, because "the spatial sense of the skin could no longer discern the coarse texture of the materials, and the vibrations necessary for the recognition of fine texture were lost as a result of the minimal friction" (p. 138).

Although never entirely lost sight of, Katz's duplex theory was somewhat overshadowed in subsequent decades, especially in the wake of landmark studies by Lederman and Taylor (1972; Taylor \& Lederman, 1975). These researchers obtained compelling evidence that the roughness of the milled gratings they used as stimuli was closely tied to their spatial properties. The speed of move-

This work was supported by NSF grant SBR-9514432. The authors thank Robert Peters for valuable discussion. The second author is now in the Department of Psychology at the University of Arizona, Tucson. Correspondence should be addressed to M. Hollins, Department of Psychology, CB\# 3270, Davie Hall, University of North Carolina, Chapel Hill, NC 27599 (e-mail: mhollins@email.unc.edu). ment of a finger across a grating had little effect on the sensation of roughness produced, a result that is hard to reconcile with a simple code based on vibration frequency. The role of vibration in texture perception was put to a direct test by Lederman, Loomis, and Williams (1982), who found that adaptation of vibrotactile channels did not reduce the perceived roughness of gratings. Although sometimes interpreted as showing that vibrotaction does not contribute to texture perception generally, the findings of Lederman et al. (1982) actually do so only in the case of relatively coarse textures, since the finest grating they used had a spatial period (i.e., groove width plus ridge width) of $630 \mu \mathrm{m}$.

What is the physiological basis for this sensitivity to the spatial properties of stimuli? A series of studies by Johnson and colleagues (summarized by Johnson \& Hsiao, 1992) has made it clear that perception of such properties (e.g., identification of raised letters) depends largely on the processing of signals from slowly adapting Type I (SA I) mechanoreceptors; from primary afferents (Phillips, Johansson, \& Johnson, 1990) to cortical neurons (Phillips, Johnson, \& Hsiao, 1988), this mechanoreceptive system demonstrates a remarkable ability to register and extract information about the spatial arrangement of features that are large enough to be individually discerned. Moreover, Connor, Hsiao, Phillips, and Johnson (1990; Connor \& Johnson, 1992) have shown explicitly that people use this system to judge the roughness of coarse surfaces and that their judgments reflect computations, apparently made in somatosensory cortex, using spatial algorithms.

In attempting to relate Katz's ideas to the perspectives developed by later workers, care must be exercised in de- 
fining and using the term spatial. Multiple definitions are possible, depending on the types of stimuli used and the psychophysical judgments required. In the context of tactile textures, "spatial" sensitivity can mean processing that results in perception (or explicit neural representation) of the size, arrangement, and other geometrical properties of texture elements. Alternatively, the term can be more broadly used to mean sensitivity to those aspects of sensory information that do not depend on temporal dynamics - what Taylor and Lederman $(1975$, p. 24), in their static analysis of touch, refer to as "the shape of the skin at any moment." These two meanings overlap but do not coincide; for example, small-scale patterns of deformation of the skin may be able to influence neural activity, and indeed perception, without being subjectively or neurally represented in spatial terms.

By either definition, it is clear that textures composed of elements too small to appreciably deform the skin must be perceived in some nonspatial way, perhaps involving vibratory activation of rapidly adapting channels (Heller, 1989; Johnson \& Hsiao, 1992; LaMotte \& Srinivasan, 1991). Thus, studies of coarse-texture perception implicate spatial processing, whereas data on perception of fine textures are at least compatible with the use of vibrational cues. What is not presently available, however, is a psychophysical demonstration, within a single study, that different mechanisms underlie the perception of fine and coarse textures.

To at least partially dissociate spatial and vibrational sources of information, the present study made use of another experimental strategy used by Katz $(1925 / 1989$, pp. 100-109): comparing the perception of texture with and without movement of the finger across the surface (or the surface across the finger). Katz reported that, without lateral movement, subjective impressions of the texture of his stimulus papers were extremely vague; only hardness and softness could still be discerned. That texture perception is enhanced by movement is an observation that has been made by many later authors as well (e.g., Morley, Goodwin, \& Darian-Smith, 1983; Taylor $\&$ Lederman, 1975). But if the duplex theory of texture perception is correct, eliminating lateral movement should interfere mainly with the perception of fine textures, because their perception requires vibration that will not occur under conditions of static touch; coarse textures ought still to be discernible, to some degree, since slowly adapting afferents can register considerable spatial information even without lateral movement (Phillips \& Johnson, 1981).

Such a dissociation between fine and coarse textures is sometimes asserted on the basis of common experience (Phillips, Johansson, \& Johnson, 1992), but there is little quantitative evidence for it other than that provided by Katz himself. For example, another early study (Meenes \& Zigler, 1923) reported that subjects who described moving textured stimuli as rough or smooth did not find these terms appropriate to describe the same surfaces when lateral movement was disallowed, preferring to describe their sensations in terms of even or uneven pressure; but there is no indication in their study that this was more true of fine textures than of coarse textures. More recent studies of roughness perception generally confine themselves to relatively coarse textures (Connor et al., 1990; Connor \& Johnson, 1992) or do not measure performance under conditions of static touch (Heller, 1989; Lamb, 1983; Lederman, 1974; Lederman \& Taylor, 1972; Stevens \& Harris, 1962; Taylor \& Lederman, 1975). Thus, Phillips, Johnson, and Browne's (1983, p. 243) prediction that "texture discrimination ... that is believed to depend on information relayed by the [vibration-sensitive] rapidly adapting afferents should be profoundly affected by skin movement" has rarely been subjected to quantitative test.

The most relevant observations are those of LaMotte and his colleagues. LaMotte and Srinivasan (1991; see also Srinivasan, Whitehouse, \& LaMotte, 1990) have found that an array of dots or bars that are on the order of $0.1 \mu \mathrm{m}$ in height can be detected by scanning, but not by static, touch. Subjects reported that their detection of these ultrafine stimuli (to which only Pacinian afferents were found to respond) was based on vibratory sensations. It is not known, however, whether the roughness of the perceived texture varies in any systematic way with the quality of these vibratory sensations.

As surfaces with increasingly large texture elements are presented, a point will eventually be reached at which the texture can be discerned by static touch-a point, in other words, at which information about "the shape of the skin at any moment" begins to influence perception. Such an influence (presumably mediated by the SA I mechanoreceptive system) would constitute spatial processing in the broad sense of the term, whether or not individual texture elements were resolvable. The stimulus dimensions at which this static information begins to contribute to texture perception are not presently known.

The purpose of the present study was to use converging operations (i.e., discrimination and scaling paradigms) to test the prediction that the elimination of lateral movement would substantially compromise the perception of fine textures, but not coarse ones. A further goal, if the experimental results supported the duplex theory, was to identify the point (if the transition were abrupt) or region (if it were gradual) on the physical continuum of texture-element size at which motion ceases to be crucial for texture perception-presumably indicating the engagement of a nonvibrational mechanism.

The present experiments dealt with a particular dimension of perceived texture-namely, roughness/ smoothness - and used as stimuli abrasive papers spanning a wide range of particle sizes. Three experiments were carried out. Experiment 1 used a forced-choice procedure to measure subjects' texture discrimination when stimulus surfaces were drawn past the fingertip and when they were held in stationary contact with it. Experiments 2 and 3 made use of free magnitude estimation to assess the relative roughness (or, in Experiment 3, properties seman- 
tically related to roughness) of textured stimuli examined with and without lateral movements.

\section{EXPERIMENT 1}

In Experiment 1, the ability to discriminate two fine surfaces (particle sizes $<20 \mu \mathrm{m}$ ) and two coarse ones $(>100 \mu \mathrm{m})$ was measured under both stationary and moving conditions. The duplex theory predicts that, other things being equal, movement should have a greater facilitating effect on the discrimination of fine surfaces than it has on the discrimination of coarse ones. A forcedchoice procedure was used so that the results would not be affected by criterion changes that might occur between the movement and no-movement conditions.

\section{Method}

Subjects. There were 8 subjects ( 1 male, 7 females), undergraduate students who responded to a flyer posted in the psychology building offering payment for participation. (This method of recruitment was used throughout the study.) The subjects ranged in age from 18 to 22 years; all were right-handed. They used the index finger of their preferred hand to touch the stimulus surfaces (as was the case in all experiments).

Apparatus and Materials. The stimulus textures were four abrasive papers: a fine pair with particle sizes of 9 and $15 \mu \mathrm{m}$, and a coarse pair with particle sizes of 141 and $192 \mu \mathrm{m}$. (These and other surfaces used in the study are described in more detail later.)

The stimulus pairs were chosen on the basis of pilot measurements made on a separate group of 8 subjects, using procedures identical to those in the present experiment. The pilot measurements showed that 9 - and $15-\mu \mathrm{m}$ papers were discriminated well under conditions of moving touch ( $M$ percent correct $=98.25, S E M=$ $0.70)$ but not static touch $(M=53.75, S E M=3.43)$, consistent with the hypothesis; a sign test showed that the effect of movement was significant $(p<.01)$. Papers with particle sizes of 116 and $192 \mu \mathrm{m}$, however, were easily discriminated under both moving $(M=97.75, S E M=0.88)$ and stationary $(M=97.25, S E M=1.06)$ conditions. The presence of a ceiling effect with these latter surfaces left unresolved the question of whether movement facilitates discrimination in this range of particle sizes. The fine surfaces used in the pilot work were therefore retained in Experiment 1 , but coarse surfaces affording a more challenging discrimination were substituted for those used in the pilot measurements.

The papers were cut into strips $15 \mathrm{~cm}$ long and $5 \mathrm{~cm}$ wide, and each strip was attached with two-sided tape to a piece of balsa approximately $15 \mathrm{~cm} \times 10 \mathrm{~cm} \times 3.5 \mathrm{~mm}$. The balsa rested on a horizontal glass plate, across which it could be smoothly drawn by the experimenter. The subject was seated at a table to which the glass plate was firmly attached; his/her forearm rested on a pillow, with the wrist and hand extending forward, palm down, onto a horizontal piece of $1.2-\mathrm{cm}$-thick styrofoam mounted several centimeters above the glass plate. The index finger was positioned over an aperture in the styrofoam, $10 \mathrm{~cm}$ long and $2 \mathrm{~cm}$ wide, $2.5 \mathrm{~cm}$ below the top of which the stimulus texture was located. By flexing this finger, the fingertip could be lowered into contact with the stimulus.

A curtain blocked the subject's view of apparatus, stimulus materials, and experimenter, and low-pass ( $<400 \mathrm{~Hz}$ ) noise ( $88 \mathrm{~dB} \mathrm{SPL})$, continuously presented through earphones, prevented the subject from hearing the faint sounds made by movement of the surfaces along the finger. An indicator light, operated by the experimenter, informed the subject when to lower his/her finger onto the textured surface (light on) and when to lift the finger back up (light off).

This experimental set-up was used in all four experiments of the study.
Procedure. Once the experimental procedures had been explained to the subject, and informed consent has been obtained, he or she was seated at the experimental table and asked to extend the preferred hand and forearm under the curtain. The subject was familiarized by touch with the styrofoam platform and was given practice in lowering and raising the finger in response to the signal light. The earphones were then put on the subject, and the experiment itself began. Two stimuli were presented on each trial. For each presentation, the subject (in response to the light) lowered the index fingertip enough to apply "gentle pressure" on the textured surface, with which it remained in contact for approximately $1 \mathrm{sec}$. During this exposure period, the balsa-mounted stimulus paper was either held motionless by the experimenter (on stationary trials) or drawn smoothly across the glass at a rate of approximately $9 \mathrm{~cm} / \mathrm{sec}$ so that it traversed the subject's index fingerpad longitudinally in a proximal-to-distal direction (on movement trials). Following an interval of about $3 \mathrm{sec}$, the second stimulus was presented in the same way. The stimuli on a trial were either the two members of the fine pair ( 9 and $15 \mu \mathrm{m})$ or the two members of the coarse pair (141 and $192 \mu \mathrm{m}$ ), and either both were moving or both were stationary. Following presentation of the second stimulus, the subject indicated, by saying "one" or "two," in which of the two stimulus intervals the rougher surface had been presented. No feedback was given. Several seconds, during which the experimenter recorded the response, intervened before the next trial.

A subject's participation consisted of four runs, each 50 trials in length and devoted to one of the four conditions in the $2 \times 2$ design (fine/coarse surfaces $\times$ movement/no movement). Order of presentation of the two stimuli within a trial was random, subject to the constraint that each came first on half of the 50 trials in the run. Separate random orders were generated for every run for every subject. The four conditions were arranged in different random orders for 4 of the subjects; the other 4 subjects received the conditions in the reverse order(s). A short break was given between successive runs.

\section{Results}

Discriminability of the fine surfaces was excellent when the surfaces were drawn across the subject's skin ( $M$ percent correct $=93.75, S E M=3.41)$ but declined to a level $(M=56.00, S E M=2.54)$ that was only marginally better than chance (sign test, $p=.016$ ) when this movement was eliminated. The effect of movement was statistically significant (sign test, $p<.01$ ), reflecting the fact that it was demonstrated by all subjects.

With coarse surfaces, the substitution of a $141-\mu \mathrm{m}$ texture for the $116-\mu \mathrm{m}$ texture used in pilot work had the desired effect of reducing discriminability to a moderate level. Importantly, however, performance remained comparable in the movement $(M=78.25, S E M=8.20)$ and no-movement $(M=78.75, S E M=4.07)$ conditions, the difference between them being statistically insignificant $(p>.05)$.

\section{Discussion}

Discriminability of the two coarse (particle size > $100 \mu \mathrm{m}$ ) textures used in this experiment was equivalent in the moving and static touch conditions. Discrimination of the fine textures, in contrast, was substantially better when moving rather than stationary stimulus presentation was employed; the close correspondence between these measurements and those obtained in pilot work demonstrate that this facilitation is a reliable phenomenon. The results of Experiment 1 thus show convinc- 
ingly that movement has an important effect on the ability to discriminate fine surfaces, but not on the ability to discriminate coarse ones.

\section{EXPERIMENT 2}

Experiment 1 showed that fine textures that are easily distinguished on the basis of roughness when they move across the finger are indiscriminable under conditions of static touch. This impaired discrimination, when movement is prevented, represents an impoverishment of perception that may manifest itself in another way as well: It is reasonable to expect that an ordered set of tactile stimuli will give rise to a smaller range of sensation magnitudes when the stimuli are difficult to discriminate (e.g., under conditions of static touch) than when they are readily discriminable (when stroked across the skin). For example, estimates of the roughness of a series of fine abrasive surfaces may be more compressed under stationary conditions than when lateral movement is allowed. Discrimination performance is not, however, an invariable predictor of the slope of a psychophysical magnitude function (Gescheider, Bolanowski, Zwislocki, Hall, \& Mascia, 1994; Zwislocki \& Jordan, 1986); an empirical test is needed to determine whether the impoverishment of texture perception that occurs when movement is prevented can also be measured using ratio scaling. We therefore undertook to determine whether the role of lateral movement in texture perception, implied by our discrimination results, could be confirmed by measurements of apparent roughness.

In Experiment 2, subjects examined a series of abrasive papers, ranging widely in particle size, and judged their roughness using free (no modulus) magnitude estimation. Estimates were obtained both with and without movements of the textured surface past the fingerpad. The duplex theory predicts that perception of fine textures should be vague and uncertain when lateral movements are not permitted, a subjective change likely to manifest itself as a decrease (in the limit, to zero) in the slope of the psychophysical magnitude function for small particle sizes.

\section{Method}

Subjects. Eight undergraduate students ( 3 males, 5 females), new to the study, participated. All subjects were between the ages of 18 and 22 years; all were right-handed. One of them replaced a subject whose data were discarded because she returned from a break prematurely and saw the stimulus materials.

Apparatus and Materials. The stimulus textures were a series of abrasive surfaces (informally, "sandpapers"), with (average) particle sizes of $9,15,30,40,50,60,80,100,116,192,300$, and $350 \mu \mathrm{m}$. This range was chosen because it extended from what we considered a fairly smooth surface $(9 \mu \mathrm{m})$ to a fairly coarse one $(350 \mu \mathrm{m})$. Sandpapers differ from one another in a variety of ways other than particle size, such as the material from which the particles are made, the shape of the particles, the nature of the paper or film to which they are attached, and the adhesive or resin used to attach them. It was not possible to obtain a set of surfaces spanning the range from 9 to $350 \mu \mathrm{m}$ in which all properties other than particle size were held constant. We instead selected a set of surfaces that, on haptic inspection, felt qualitatively similar except for roughness, which gradually and montonically increased with particle size. The eight finest papers were made with aluminum oxide on a plastic film backing (3M Imperial Microfinishing Film); the 116and $192-\mu \mathrm{m}$ stimuli were garnet on a paper backing (Norton, Product Nos. A513 and A514, respectively), and the two coarsest were aluminum oxide on paper (3M, Product Nos. $240 \mathrm{~N}$ and $346 \mathrm{U}$ ).

It is worth noting that manufacturers are increasingly tending to specify the grade of abrasive surfaces by particle size ("micron grade") in addition to, or even instead of, the traditional "grit number." This trend is fueled by the fact that particle size refers to a property of the abrasive elements themselves, whereas grit number refers only to a step in the (increasingly complex) process of abrasive manufacture - that is, "the number of openings per inch in the screen employed to sift the abrasive particles" (Stevens \& Harris, 1962, p. 489). Grit number is not strictly proportional to the reciprocal of particle size, because some space is taken up by the components of the screen itself; our comparison of manufacturer-supplied grit numbers and particle sizes for the same surfaces (3M Superabrasives and Microfinishing Systems Product Guide, 1994) shows that in fact the $\log$ of grit number increases at only $84 \%$ of the rate at which $\log$ particle size decreases. The grit numbers of the surfaces used in Experiments 2 and 3 were (in order of increasing coarseness) $1200,600,400,320,240,220,180,150,120,80,50$, and 36.

Three of these surfaces were identical to those used in Experiment 1, but the $141 \mu \mathrm{m}$ surface in Experiment 1 (Norton, Grit No. 100, garnet on paper, Product No. A511) was not included in the present series.

Procedure. Each subject was tested in a single session lasting about $1 \mathrm{~h}$. After the procedure was described, the subject gave written informed consent and then was escorted into the experimental room and seated at the table. Next, instructions regarding magnitude estimation were given, as follows:

\footnotetext{
What we want you to do is to give magnitude estimates of the perceived roughness of surface textures. Magnitude estimates are numbers that you think represent the roughness that you feel on a given surface; so the rougher the surface, the higher the number. For instance, suppose you are presented with a surface and you give an estimate of its roughness; then you feel another surface that is twice as rough. You should give it a number that is twice as much as the estimate given for the first surface. Use any numbers that you think best represent your perception of the roughness. You may use whole numbers, decimals, and fractions. These numbers can be as large or as small as you want
}

Once the subject demonstrated verbal understanding of the magnitude estimation task, the experiment began. Method of stimulus presentation was the same as in Experiment 1, except that only a single stimulus presentation occurred on each trial. After lifting the finger off the stimulus, the subject verbally gave his/her magnitude estimate of its perceived roughness. Several seconds (during which the experimenter recorded this response and put the next stimulus surface in place) intervened before the next trial.

Within a block of 24 trials, each of the 12 stimulus surfaces was presented twice: once on a stationary trial and once on a movement trial. These 24 trials were ordered randomly. In all, six such blocks were carried out on each subject; a 5-min break was given after every second block. A different random order of trials was used for each block for each subject. The first of the six blocks was considered practice and was not included in the data analysis.

\section{Results}

To compute for each subject the mean log magnitude estimate given to each stimulus under each of the two experimental conditions, it was first necessary to replace 
occasional "zero" responses; this was done by substituting for zero a number half the size of the smallest nonzero estimate given by that subject to any stimulus on any trial. All data were then converted into (base-10) logarithmic form. Means of the log roughness judgments are plotted in Figure 1, as a function of particle size (scaled logarithmically). To permit assessment of subject-to-subject differences in the shape, rather than the vertical position, of these logarithmic functions, each subject's log estimates were incremented or decremented by the amount needed to make their average equal to the grand mean across subjects; error bars $( \pm 1 S E M)$ reflect the variability of these normalized data.

Open circles show the results obtained when the surface was moved across the skin, and filled circles show the responses given when movement was not permitted. The function for the stationary condition is somewhat below the movement function over most of the stimulus range, but the two intersect between 15 and $30 \mu \mathrm{m}$. The two functions also differ in shape: the movement function is approximately linear over the entire range of particle sizes in this $\log -\log$ plot, but the function for the stationary condition is concave upward, roughly paralleling the movement function for particle sizes above $100 \mu \mathrm{m}$ but becoming nearly horizontal for finer surfaces. Because the relationship between the functions appears to be different above and below $100 \mu \mathrm{m}$, we compared the increase in roughness from 9 to $100 \mu \mathrm{m}$ in the stationary condition with that in the moving condition and (separately) made an analogous comparison with respect to the further increase in roughness from 100 to $350 \mu \mathrm{m}$. Although opposite in direction, the differences between stationary and moving conditions were significant in both portions of the stimulus continuum [for coarse surfaces, $t(7)=$ $5.70, p<.01$; for fine surfaces, $t(7)=-5.03, p<.01]$.

\section{Discussion}

Eliminating lateral movement between stimulus surfaces and the fingertip had two salient effects: a reduction in the slope of the psychophysical magnitude function for fine surfaces, and an overall decline in roughness (combined with a modest increase in slope) for coarse surfaces. Movement increased the roughness of most of the surfaces employed, but this overall increase does not by itself imply an enrichment or improvement of texture perception. The ability consistently to assign different textural values to different surfaces (i.e., slope of the psychophysical magnitude function) is a more useful index of the sensory information obtained, and, by this measure, texture perception was not at all impoverished by the elimination of movement for surfaces with particle sizes $>100 \mu \mathrm{m}$. For the finer surfaces (particle size $<100 \mu \mathrm{m}$ ), however, lack of movement largely deprived the subjects of the ability to experience the nearly threefold increase in roughness, as particle size increased from 9 to $100 \mu \mathrm{m}$, that they reported when movement occurred. In summary, elimination of movement produced changes across the stimulus continuum, but these changes constituted an

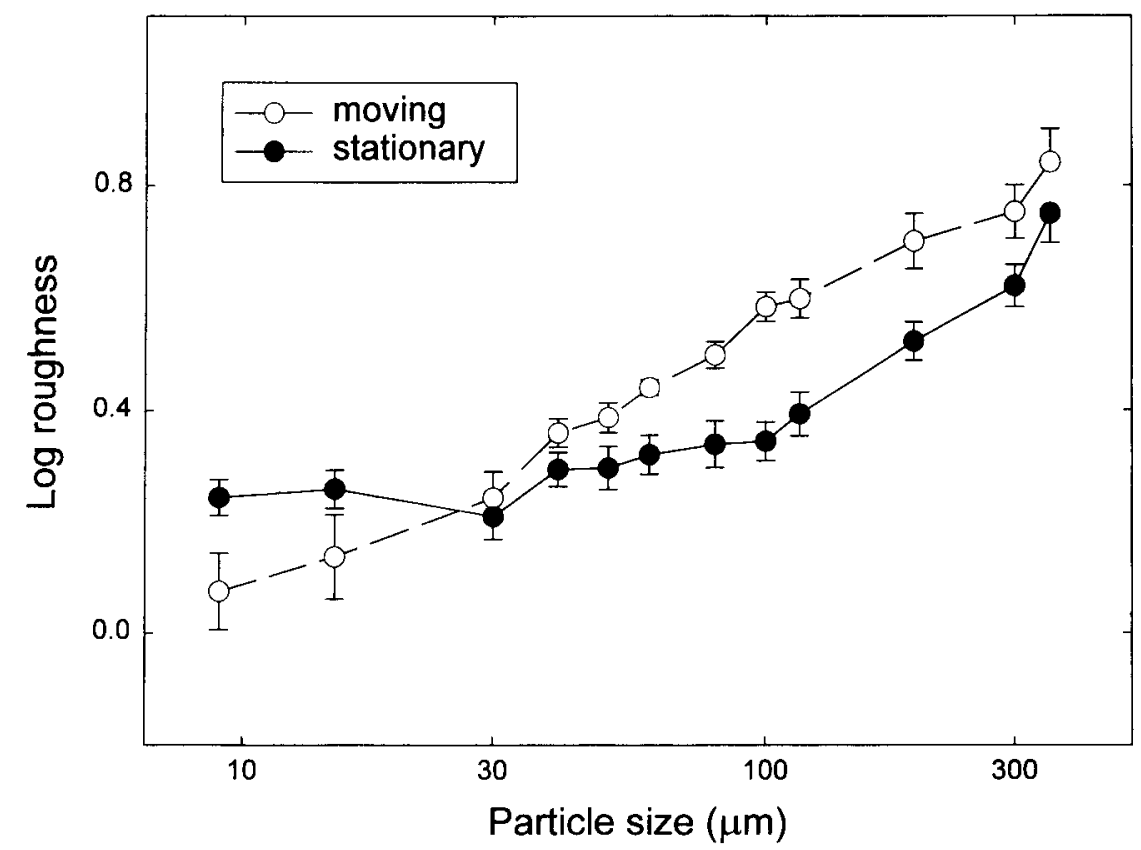

Figure 1. Log-estimated roughness in Experiment 2, plotted as a function of the particle sizes of the abrasive textures. Each point represents the mean $\log$ value across subjects $(N=8)$, under conditions of movement (open symbols) and static touch (filled symbols). Data were normalized across subjects as described in the text before $S E M$ s (error bars) were calculated. Overlapping error bars are omitted for clarity. 
impoverishment of perception only for fine surfaces. The results are thus consistent with the predictions of the duplex theory of texture perception.

A potential caveat to this conclusion concerns the scale on which the subjects were asked to make their ratingsa scale of roughness. In discussing the experiment after their participation had ended, the subjects sometimes commented that it was difficult to assess the roughness of the finer surfaces in the no-movement condition. It occurred to the experimenters that the subjects' difficulty in making these judgments may have arisen not from the fact that the stimuli were not clearly perceived but from the fact that the subjects felt the term roughness no longer accurately captured their sensory experience. Exactly this sense of the inappropriateness of the terms rough and smooth was reported by the subjects in Meenes and Zigler's (1923) study, who preferred to use the terms even and uneven (pressure) to describe their sensations when movement across the textured surfaces was not allowed. Perhaps, in the absence of movement, the finer surfaces in the present experiment are likewise distributed along some sensory dimension other than roughness, a dimension that the procedure did not allow the subjects to report on. It was to address this possibility that Experiment 3 was undertaken.

\section{EXPERIMENT 3}

Experiment 3 was identical to Experiment 2, except for the selection of the scale on which subjects were to rate the stimuli. The purpose of the experiment was to carry out a strong test of the possibility that, under conditions of static touch, subjects perceive differences among the fine textures, but experience these as different amounts of some property other than roughness. We therefore allowed them to identify a property or properties other than roughness that distinguished one surface from another. Each subject was then asked to assess, using magnitude estimation, the amount of this property that characterized each of the stimuli.

\section{Method}

Subjects. Eight new subjects ( 2 males, 6 females), undergraduates ranging in age from 18 to 22 years, participated in this experiment. All were right-handed.

Procedure. The experimental set-up and stimulus set were the same as in Experiment 2. To select the nominal dimension on which a given subject was to be asked to rate the stimuli, he or she was first presented with two of the stimuli, those with particle sizes of 15 and $300 \mu \mathrm{m}$ (i.e., the stimuli adjacent in the series to those with the smallest and largest particle sizes, respectively). These were presented, once each, under conditions identical to those used in the stationary condition in Experiment 2. The subject was then asked whether he/she could tell the difference between the two; every subject answered in the affirmative. The subject was then asked to explain the difference by naming a property on which the two stimuli differed. If the subject said "roughness," he or she was asked to use a different term to denote the difference. All subjects offered multiple terms: interestingly, 7 subjects mentioned smoothness (specifying that the $15-\mu \mathrm{m}$ stimulus was smoother), whereas only 5 men- tioned roughness (specifying that the $300-\mu \mathrm{m}$ surface was the rougher of the two). Asked to use a descriptor other than "rough," these latter subjects said the stimuli differed in "amount of texture" or that the $300-\mu \mathrm{m}$ surface was more "raised," had more "regularity," or was "grainier," "lumpy," or "scratchy." From the terms offered by each subject, the experimenters selected one, on which that subject was subsequently asked to rate all the stimuli when presented under both stationary and moving conditions. The choice of terms was somewhat arbitrary and subject who to the constraint that 4 subjects use "smooth" or a similar term, whereas the other 4 subjects use a term similar to "rough." In the former group were 3 subjects who rated smoothness, and 1 subject who rated glassiness, a synonym she had spontaneously offered. In the latter group, the subjects were asked to rate the stimuli on the properties graininess ( 2 subjects), amount of texture (1 subject) or Velcro-likeness ( 1 subject).

Once the choice of term had been made and communicated to the subject, the experiment proceeded according to the same protocol as Experiment 2.

\section{Results}

When asked to rate the stimuli in terms of the designated property, the subjects gave ratings that tended either to increase steadily (in the case of words similar in meaning to roughness) or to decrease steadily (in the case of the words smoothness and glassiness), with increasing particle size. Data from the subjects who gave ratings on a property similar to roughness are shown in Figure 2. These functions are similar to the roughness estimates from Experiment 2 (Figure 1). The subjects in the two experiments appear to be judging amounts of closely related sensory properties or (more likely) the same property, whether it is denoted by roughness or by another term similar in meaning; the data suggest that the underlying sensory dimension is a robust and salient one that transcends variation in terminology.

Data from the subjects who used the terms smoothness or glassiness to rate the same stimuli are shown in Figure 3. If smoothness and roughness were reciprocally related, then it would be expected that these functions would resemble inverted transformations of the functions shown in Figures 1 and 2. The general trend of the data is in the appropriate direction - smoothness decreases as particle size increases-but there is a clear difference in shape between the smoothness and roughness functions: For particle sizes greater than $100 \mu \mathrm{m}, \log$ roughness increases at a fairly steady rate (Figures 1 and 2 ), whereas $\log$ smoothness decreases more and more rapidly (Figure 3 ).

Despite this difference between estimates of roughness and smoothness, the two sets of data are consistent with respect to the way sensory judgments are affected by the elimination of movement: In both cases, the magnitude estimation functions grow shallower for finer surfaces, whereas they actually grow somewhat steeper for coarse surfaces (those with particle sizes $>100 \mu \mathrm{m}$ ).

\section{Discussion}

These results argue that elimination of lateral movement at the skin-stimulus interface actually degrades texture perception, rather than merely causing a qualitative change so that the stimuli are perceived as ranged along 


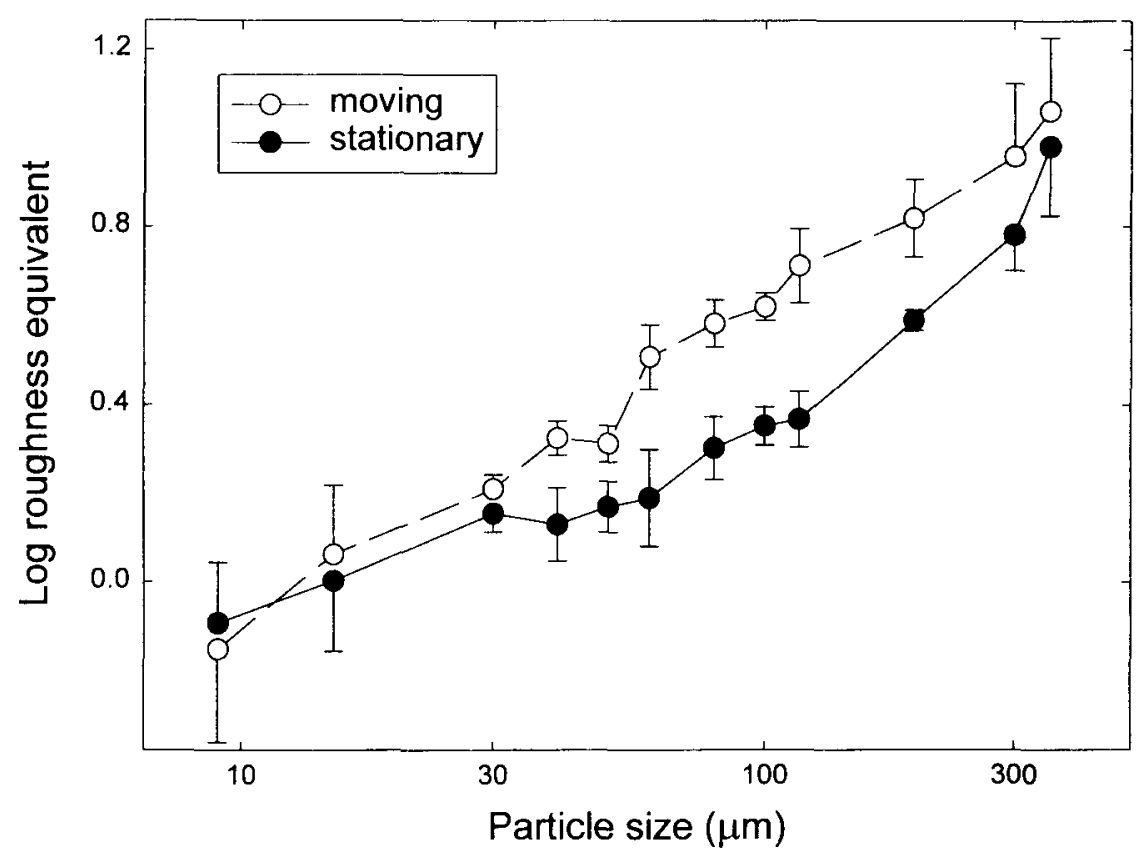

Figure 2. Log magnitude estimates obtained in Experiment 3 when subjects $(N=4)$ rated the surfaces on the extent to which they displayed properties semantically equivalent to roughness, such as graininess. Open and filled symbols represent data from conditions of movement and static touch, respectively. Data were normalized across subjects as described in the text before $S E M$ s (error bars) were calculated. Overlapping error bars are omitted for clarity.

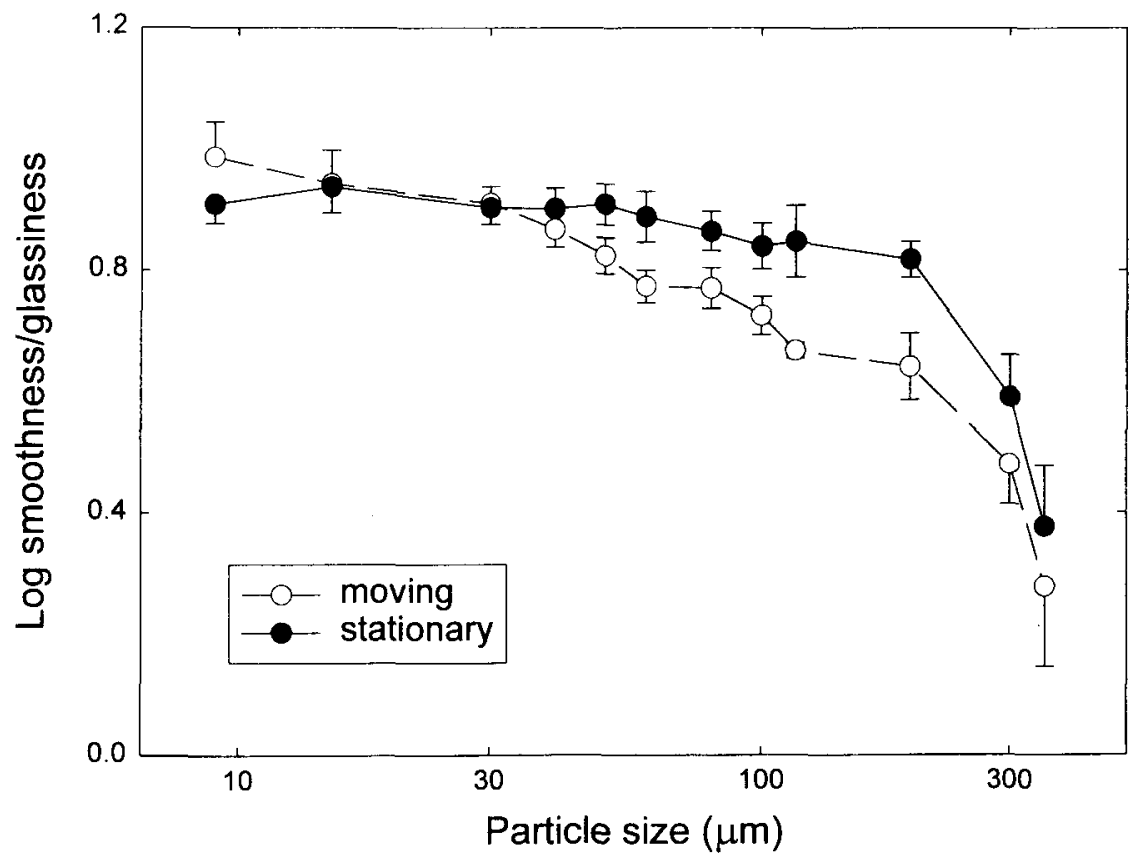

Figure 3. Log magnitude estimates obtained in Experiment 3 when subjects $(N=4)$ judged the smoothness or glassiness of the surfaces. Open and filled symbols represent data from conditions of movement and static touch, respectively. Data were normalized across subjects as described in the text before $S E M$ s (error bars) were calculated. Overlapping error bars are omitted for clarity. 
a different dimension. Even when allowed to rate the stimuli on some property of their own choosing (other than roughness), the subjects' responses indicated a flattening of the psychophysical magnitude function over the "fine" $(<100 \mu \mathrm{m})$ portion of the particle size continuum. No such reduction in slope occurred for coarser surfaces, however. The data thus give further support to the duplex theory.

The difference in shape of the functions for roughness/ graininess (etc.) on the one hand and smoothness/glassiness on the other hand (Figures 1, 2, and 3) was somewhat surprising, in view of the finding by Stevens and Harris (1962) that magnitude estimates of roughness and smoothness are reciprocally related. They asked subjects to rate both the roughness and (in a separate session) the smoothness of each of a series of 12 emery cloths ranging in grit number from 320 to 24 (equivalent to particle sizes of 40 and $\sim 820 \mu \mathrm{m}$ ), examined by active touch. When (as in the present study) no modulus was used, subjects' responses were well described by power functions. The main discrepancy between our findings and those of Stevens and Harris is that our smoothness function was curvilinear in $\log -\log$ coordinates, with subjects giving very low smoothness ratings to the $2-3$ coarsest surfaces. One possible explanation for this deviation from the power law is that the smoothness of the finest surfaces in the present study (having smaller particles than those used by Stevens and Harris) was a property so qualitatively distinctive that it seemed to be absent from the coarsest surfaces - that the subjects in the present study, in other words, allowed that the finest surfaces possessed at least a little roughness but that the coarsest ones possessed virtually no smoothness. An alternative hypothesis to explain the difference in results between the two studies is that Stevens and Harris's subjects, rating both roughness and smoothness, came to appreciate the possibility that a simple relationship (reciprocality) might exist between these two properties. In the present study, separate groups of subjects rated roughness and smoothness, so the likelihood was reduced that they would give estimates of smoothness that were, in Stevens and Harris's (1962, pp. 492-493) words, "inversely proportional to those he [or she] would use for the continuum 'right side up' ... provided he [or she] understands the semantic rule: a surface that is twice as rough is half as smooth."

The two functions in the present study that are reasonably linear in log-log coordinates, the unadapted roughness (Figure 1) and roughness-like (Figure 2) functions, have slopes of 0.49 and 0.74 , respectively. These functions are considerably shallower than that found by Stevens and Harris (with a slope of approximately -1.5 ), but it is not obvious which of the many differences between the studies (active vs. passive stimulation, qualitatively different surfaces, a larger stimulus range in the present study, etc.) account(s) for the difference. Complicating a comparison is the fact that Stevens and Harris denoted their surfaces by grit number, whereas we used particle size; as explained earlier, grit number varies as a power function (with exponent $=-.84$ ) of particle size. The fact that the relationship between these two quantities is not a strictly reciprocal one accounts for some of the difference in absolute value of slope between our roughness functions and that of Stevens and Harris, but most of the difference remains to be explained.

Whatever the correct interpretation of this difference between studies, it does not affect the major finding of Experiments 2 and 3, which is that, whether subjects rate roughness or smoothness, movement increases the slope of the magnitude function for surfaces with particle sizes less than, but not greater than, $100 \mu \mathrm{m}$.

\section{GENERAL DISCUSSION}

The three experiments of the present study make it clear that lateral movement between the skin and a textured surface has dramatically different effects on the perception of fine surfaces and the perception of coarse ones. For textures composed of particles smaller than $100 \mu \mathrm{m}$, eliminating movement constricted the subjective range over which roughness (Experiment 2) or other sensory qualities said by the subjects to characterize the surfaces (Experiment 3 ) were distributed; in contrast, coarse surfaces were perceived as less rough in the absence of movement, but the logarithmic range over which magnitude estimates were distributed was, if anything, slightly increased. Consistent with these scaling findings, discrimination of fine surfaces was markedly better with lateral stimulus movement than without it, whereas the discrimination of coarse surfaces was unaffected (Experiment 1).

These results are entirely consistent with the duplex theory of texture perception, according to which perception of fine textures is attributable largely to vibrations set up by the relative movement of skin and stimulus, whereas coarse textures are perceived largely on the basis of their geometrical properties (when temperature, hardness, etc., are held constant). The withholding of experimenter-produced stimulus movement substantially impairs perception of fine textures because it nearly eliminates vibrational cues; presumably, the only remaining vibration is that caused by involuntary micromovements of the finger caused (e.g.) by muscle tremor or the pulse. Small, unnoticed movements of this type may be what kept static-touch discrimination of the fine surfaces slightly above chance in Experiment 1 and what prevented the slope of the psychophysical magnitude function from dropping entirely to zero in the stationary condition of Experiments 2 and 3.

In the present study, movement did not increase the ability to discriminate between coarse stimuli. This is compatible with the idea (Taylor \& Lederman, 1975) that roughness is based on static information (spatial in the broad sense of the term), since performance on tasks that are unambiguously spatial, such as pattern recognition, have been shown to be only modestly improved by movement-even active movement in which the subject controls direction and velocity (Phillips et al., 1983). Although movement did not facilitate the discrimination of 
coarse surfaces in the present study, it did increase their subjective roughness (Experiments 2 and 3 ). This may be related to the fact that movement produces as much as a tenfold increase in the activity of afferents in the SA I channel (the channel primarily responsible for encoding spatial information; Johnson \& Hsiao, 1992) when they are stimulated by coarse spatial arrays (Johnson \& Lamb, 1981). Could movement trigger an even greater amplification of spatial signals in the case of fine surfaces and thus account for their enhanced discriminability in the present study? Although this possibility cannot be conclusively eliminated, it seems unlikely in view of the fact that the finest surfaces were perceived as smoother when moving than when experienced with static touch.

In regard to the issue of whether movement enhances spatial processing, it is instructive to consider the findings of Morley et al. (1983), who compared grating discrimination under conditions of active movement and static touch. Their standard grating had a spatial period of $1 \mathrm{~mm}$, which is coarse enough to engage spatial mechanisms. Discrimination was moderately impaired when movement was prevented, although it was, in the words of the investigators, "still impressive" (p. 298). This result differs from the finding in the present study that discrimination of coarse textures was unaffected by movement. There were several differences in procedure between the two studies that could explain the different findings. For example, Morley et al. allowed subjects to examine the stimuli repeatedly and at length (up to $10 \mathrm{sec}$ per trial); no attempt was made to equate examination times in their movement and no-movement conditions. The most likely explanation, however, is that their gratings, being spatially periodic, gave rise during movement but not during static touch to mechanoreceptor activity characterized by strong temporal periodicities (Darian-Smith \& Oke, 1980). Although temporal cues do not appear to contribute to the roughness of coarse textures in a scaling task (Connor et al., 1990; Connor \& Johnson, 1992), they might be utilized in a discrimination task if spatial information were marginal. Because of the haphazard arrangement of particles on abrasive surfaces, temporal periodicity was not a factor in the present study, perhaps resulting in a crisper transition between spatial and nonspatial mechanisms of texture perception.

One of the most intriguing results of the present study is that the transition between the portion of the roughness magnitude function that is made steeper by movement and the portion whose slope is reduced by movement occurs at about $100 \mu \mathrm{m}$. This is illustrated in Figure 4, in which the log change in perceived texture attributable to movement is plotted as a function of particle size. For Experiment 2 and for the component of Experiment 3 in which the subjects rated the surfaces on a property semantically equivalent to roughness, the plotted points were obtained by subtracting mean log magnitude estimates in the static condition from those in the moving condition; the converse subtraction was performed in the case of the smoothness estimates (Experiment 3). The

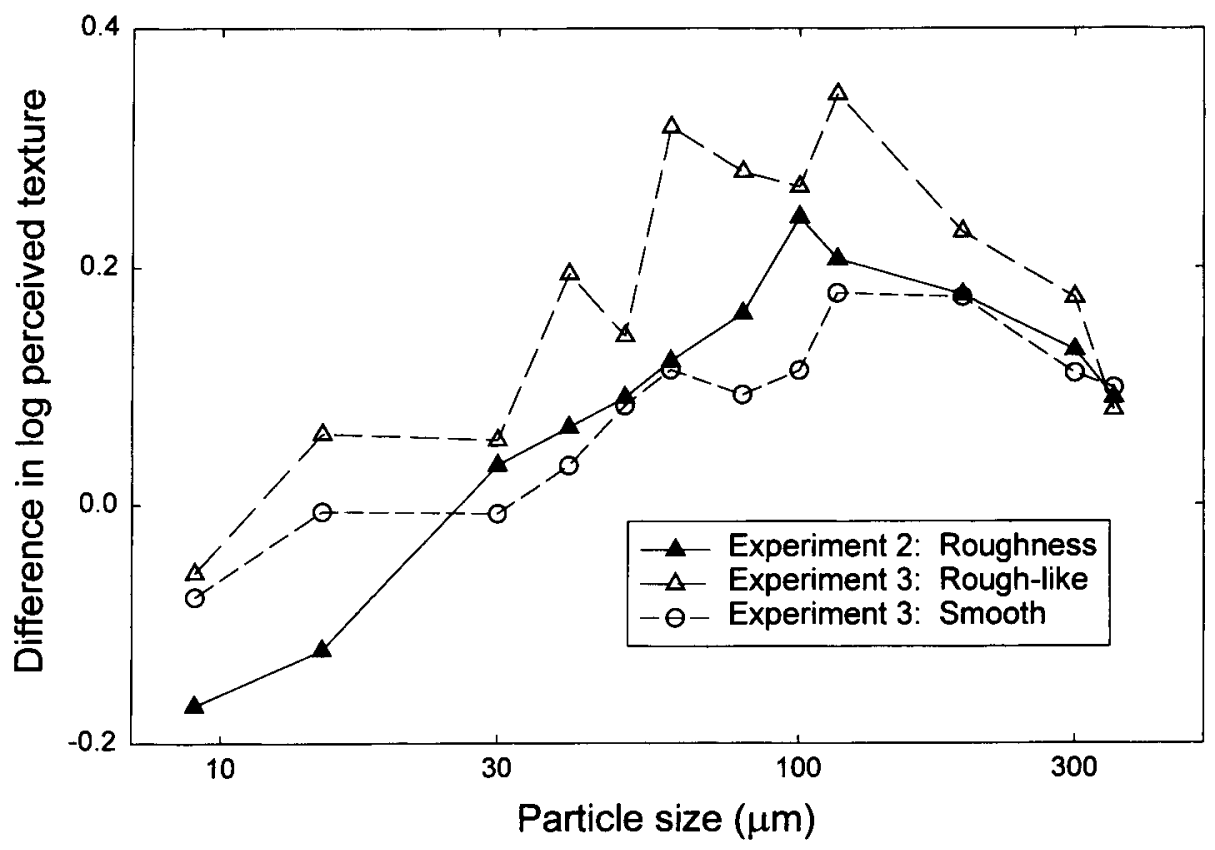

Figure 4. Difference between log magnitude estimates in the moving and stationary conditions, as a function of particle size. Positive values on the ordinate indicate that, during movement, a surface was perceived as rougher (triangles) or less smooth (circles) than during static touch. Negative values at the lowest particle sizes suggest that these fine surfaces felt very smooth when they were moved across the finger but did not evoke clear textural sensations during static touch. Filled and open symbols represent data from Experiments 2 and 3, respectively. 
shapes of the three curves are in reasonable agreement, all rising to a maximum in the vicinity of $100 \mu \mathrm{m}$ before declining slightly at still larger particle sizes.

These functions are readily interpretable in terms of the duplex theory: For fine surfaces (below $100 \mu \mathrm{m}$ ), vibration is the main cue to texture, and it indicates increasing roughness with increasing particle size-hence the rising left slope of the function. Above $100 \mu \mathrm{m}$, spatial mechanisms are progressively engaged and gradually become the dominant contributor to texture perception. Because they are relatively indifferent to movement, the functions decline as particle size continues to increase.

The idea that the segment above this transition represents the activity of spatial mechanisms may seem incompatible with the fact that, by several measures, spatial processing does not extend to such small dimensions. For example, under conditions of static touch, gaps less than $700 \mu \mathrm{m}$ cannot be detected, and gratings can be discriminated on the basis of orientation only if they have ridges or grooves at least $500 \mu \mathrm{m}$ wide (Johnson \& Phillips, 1981); a comparable resolution limit is suggested by data on the identification of embossed letters (Johnson \& Phillips, 1981; Phillips et al., 1983). However, Lederman's work indicates that spatial coding may determine roughness down to smaller element sizes: Using gratings with grooves as small as $175 \mu \mathrm{m}$, Lederman (1974) found that roughness was independent of movement speed, a result that appears more compatible with spatial coding than with vibrotactile coding; and the Taylor and Lederman (1975) model appears to account in spatial terms for grating roughness at groove widths down to $125 \mu \mathrm{m}$, although imperfections in the gratings introduce uncertainty into the model with stimuli this fine. It should be noted that the spatial property of stimulation that best predicted roughness in their model was total volumetric displacement of the skin from its resting configuration. While it may be expected that this property will affect the firing rate of SA I fibers, it will not be represented in a spatial way in the aggregate neural response. The overall conclusion therefore appears to be that spatial properties of stimulus elements between 100 and $500 \mu \mathrm{m}$ can contribute to textural judgments, but not to explicitly spatial judgments such as letter identification. The convergence of evidence that feature dimensions substantially less than $500 \mu \mathrm{m}$ can affect the response of spatial mechanisms to both gratings (Taylor \& Lederman, 1975) and sandpapers (the present study) should be interpreted cautiously, however, since these two types of stimuli are qualitatively different with regard to the shape and spacing of texture elements.

It has still not been directly demonstrated that vibration is the major information source for evaluating fine textures. The present study shows that movement gives rise to some important set of cues for surfaces with elements smaller than about $100 \mu \mathrm{m}$, but whether those cues are vibrational (as seems likely) or of a fundamentally different character remains to be conclusively determined. The main alternative candidate would appear to be frictional resistance. Taylor and Lederman (1975) showed, through the use of a lubricant, that friction makes little contribution to the roughness of coarse surfaces. For fine surfaces, friction and vibration are not so easy to separate experimentally. Katz (1925/1989, p. 115) found that when vibrations were eliminated but some information about frictional resistance was retained, by examining fine surfaces (papers) using a rod wrapped in cloth, only very crude texture discrimination was possible. But precise judgments of frictional resistance often depend on detection of slip (Johansson \& Westling, 1987), which, for a finely textured surface, can include explicit vibratory sensations (Srinivasan et al., 1990). Moreover, vibrations resulting from movement of textured surfaces across the skin have been recorded (Hollins, Delemos \& Goble, 1996) and found to vary with the character of the surface. There is thus reasonably convincing circumstantial evidence that vibration does make a major contribution to the perception of fine textures.

Whatever the eventual verdict regarding vibration, however, the present study shows conclusively that movement produces a dissociation in psychophysical performance between coarse and fine surfaces, at least as regards roughness judgments. The results provide evidence against theories that attribute perception of all textures to a single mechanism: Two mechanisms-one movementsensitive, the other not-appear to be needed to account for perception across the full range of discriminable textures. Katz's (1925/1989) insightful appreciation of the duplex nature of tactile texture perception is thus confirmed.

\section{REFERENCES}

Connor, C. E., Hsiao, S. S., Phillips, J. R., \& Johnson, K. O. (1990). Tactile roughness: Neural codes that account for psychophysical magnitude estimates. Journal of Neuroscience, 10, 3823-3836.

Connor, C. E., \& Johnson, K. O. (1992). Neural coding of tactile texture: Comparison of spatial and temporal mechanisms for roughness perception. Journal of Neuroscience, 12, 3414-3426.

DaRIAN-Smith, I., \& OKE, L. E. (1980). Peripheral neural representation of the spatial frequency of a grating moving across the monkey's fingerpad. Journal of Physiology, 309, $117-133$.

Gescheider, G. A., Bolanowski, S. J., Zwislocki, J. J., Hall, K. L., \& MASCIA, C. (1994). The effects of masking on the growth of vibrotactile sensation magnitude and on the amplitude difference limen: A test of the equal sensation magnitude-equal difference limen hypothesis. Journal of the Acoustical Society of America, 96, 1479-1488.

Heller, M. A. (1989). Texture perception in sighted and blind observers. Perception \& Psychophysics, 45, 49-54.

Hollins, M., Delemos, K. A., \& Goble, A. K. (1996). Vibrotactile adaptation of the RA system: A psychophysical analysis. In $O$. Franzén, R. S. Johansson, \& L. Terenius (Eds.), Somesthesis and the neurobiology of the somatosensory cortex (pp. 101-111). Basel: Birkhäuser Verlag.

JohansSON, R. S., \& WESTLING, G. (1987). Signals in tactile afferents from the fingers eliciting adaptive motor responses during precision grip. Experimental Brain Research, 66, 141-154.

JoHNSON, K. O., \& HsıAO, S. S. (1992). Neural mechanisms of tactual form and texture perception. Annual Review of Neuroscience, 15, 227-250.

Johnson, K. O., \& LAMB, G. D. (1981). Neural mechanisms of spatial tactile discrimination: Neural patterns evoked by Braille-like dot patterns in the monkey. Journal of Physiology, 310, 117-144.

Johnson, K. O., \& PhILliPs, J. R. (1981). Tactile spatial resolution: I. 
Two-point discrimination, gap detection, grating resolution, and letter recognition. Journal of Neurophysiology, 46, 1177-1191.

KATZ, D. (1989). The world of touch (L. E. Krueger, trans.). Hillsdale, $\mathrm{NJ}$ : Erlbaum. (Original work published 1925)

LAMB, G. (1983). Tactile discrimination of textured surfaces: Psychophysical performance measurements in humans. Journal of Physiology, 338, 551-565.

LaMotTe, R. H., \& SRinivasan, M. A. (1991). Surface microgeometry: Tactile perception and neural encoding. In O. Franzén \& J. Westman (Eds.), Information processing in the somatosensory system (pp. 49-58). New York: Stockton.

LEDERMAN, S. J. (1974). Tactile roughness of grooved surfaces: The touching process and the effects of macro- and microsurface structure. Perception \& Psychophysics, 16, 385-395.

Lederman, S. J., LoOmis, J. M., \& Williams, D. A. (1982). The role of vibration in the tactual perception of roughness. Perception \& Psvchophysics, 32, 109-116.

Lederman, S. J., \& Taylor, M. M. (1972). Fingertip force, surface geometry, and the perception of roughness by active touch. Perception \& Psychophysics, 12, 401-408.

MeEnES, M., \& Zigler, M. J. (1923). An experimental study of the perceptions roughness and smoothness. American Journal of Psychology, 34, 542-549.

3M Superabrasives and Microfinishing Systems Department. (1994). Superabrasives and microfinishing systems: Product guide. St. Paul, MN: Author.

Morley, J. W., Goodwin, A. W., \& Darian-Smith, I. (1983). Tactile discrimination of gratings. Experimental Brain Research, 49, 291-299.

Phillips, J. R., Johansson, R. S., \& Johnson, K. O. (1990). Repre- sentation of Braille characters in human nerve fibers. Experimental Brain Research, 81, 589-592.

Phillips, J. R., Johansson, R. S., \& Johnson, K. O. (1992). Responses of human mechanoreceptive afferents to embossed dot arrays scanned across fingerpad skin. Journal of Neuroscience, 12, 827-839.

Phill.ips, J. R., \& Johnson, K. O. (1981). Tactile spatial resolution. II. Neural representation of bars, edges, and gratings in monkey primary afferents. Journal of Neurophysiology, 46, 1192-1203.

Phillips, J. R., Johnson, K. O., \& Browne, H. M. (1983). A comparison of visual and two modes of tactual letter resolution. Perception \& Psychophysics, 34, 243-249.

Phillips, J. R., Johnson, K. O., \& Hsiao, S. S. (1988). Spatial pattern representation and transformation in monkey somatosensory cortex. Proceedings of the National Academy of Sciences, 85, 1317-1321.

Srinivasan, M. A., Whitehouse, J. M., \& LaMotte, R. H. (1990). Tactile detection of slip: Surface microgeometry and peripheral neural codes. Journal of Neurophysiology, 63, 1323-1332.

STEVENS, S. S., \& HARRIS, J. R. (1962). The scaling of subjective roughness and smoothness. Journal of Experimental Psychology, 64, 489-494.

Taylor, M. M., \& Lederman, S. J. (1975). Tactile roughness of grooved surfaces: A model and the effect of friction. Perception \& Psychophysics, 17, 23-36.

ZWISLOCKI, J. J., \& JORDAN, H. N. (1986). On the relations of intensity jnd's to loudness and neural noise. Journal of the Acoustical Society of America, 79, 772-780.

(Manuscript received July 21,1998 ; revision accepted for publication May 6, 1999.) 